\title{
A Study Examining the Effects of Tissue Processing on Human Tissue Sections using Vibrational Spectroscopy
}

\author{
Eoghan O'Faolain \\ Technological University Dublin \\ Mary Hunter \\ National Maternity Hospital, Holles Street \\ Joe Byrne \\ National Maternity Hospital, Holles Street
}

See next page for additional authors

Follow this and additional works at: https://arrow.tudublin.ie/materart

Part of the Life Sciences Commons

\section{Recommended Citation}

O'Faolaoin, E., Hunter, M. \& Byrne, J. (2005). A study examining the effects of tissue processing on human tissue sections using vibrational spectroscopy. Vibrational Spectroscopy vol. 38, Issues 1-2, pg. 121-127. 29 July. doi:10.1016/j.vibspec.2005.02.013

This Article is brought to you for free and open access by the Materials Synthesis and Applications at ARROW@TU Dublin. It has been accepted for inclusion in Articles by an authorized administrator of ARROW@TU Dublin. For more information, please contact

arrow.admin@tudublin.ie, aisling.coyne@tudublin.ie, gerard.connolly@tudublin.ie.

Funder: Technological University, Higher Education Authority

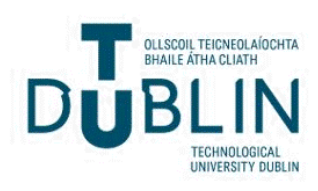


Authors

Eoghan O'Faolain, Mary Hunter, Joe Byrne, Peter Kellehan, Mary McNamara, Hugh Byrne, and Fiona Lyng

This article is available at ARROW@TU Dublin: https://arrow.tudublin.ie/materart/13 
A study examining the effects of tissue processing on human tissue sections using vibrational spectroscopy

Eoghan O'Faolaoin a, ${ }^{*}$, Mary B. Hunter b,1, Joe M. Byrne b,1, Peter Kelehan c,2, Mary McNamara d,3, Hugh J. Byrne e,4, Fiona M. Lyng f,5

a School of Physics/Focas Institute, Dublin Institute of Technology, Kevin Street, Dublin 8 , Ireland

b Department of Histology, National Maternity Hospital, Holles Street, Dublin 2, Ireland

c Department of Pathology and Laboratory Medicine, National Maternity Hospital, Holles Street, Dublin 2, Ireland

d School of Chemistry/Focas Institute, Dublin Insititute of Technology, Kevin Street, Dublin 8, Ireland

e Focas Institute, Dublin Institute of Technology, Kevin Street, Dublin 8, Ireland

f Radiation and Environmental Science Centre (RESC), Focas Institute, Dublin Institute of Technology, Kevin Street, Dublin 8, Ireland

Received 29 October 2004; accepted 14 February 2005

Available online 23 March 2005

\begin{abstract}
The use of vibrational spectroscopy in the detection of cancer is a newly emerging diagnostic field, which has shown great potential to date. Many investigations have been carried out on frozen tissue samples, which by their very nature are hard to obtain. However, histology departments have archives of thousands of tissue samples, preserved and mounted in wax blocks. If this archival material can be shown to yield good Raman and IR spectra capable of differentiating between normal and cancerous tissue, it would improve the diagnostic capabilities of spectroscopy even further. Results from these formalin-fixed paraffin processed (FFPP) tissue sections, will give a better understanding of the effects of processing and could unlock the potential diagnostic capabilities of FFPP sections. This study investigated the effect of freezing, formalin fixation, wax embedding and de-waxing. Spectra were recorded from parallel tissue sections of placenta to examine biochemical changes before, during and after processing with both Raman and IR spectroscopy. FFPP sections were shown to provide good quality Raman and IR spectra but new peaks due to freezing and formalin fixation as well as shifts in the amide bands resulting from changes in protein conformation and possible cross-links were found. Residual wax peaks were observed clearly in the Raman spectra. In the FT-IR spectra a single wax contribution was seen which may contaminate the characteristic $\mathrm{CH} 3$ deformation band in biological tissue. This study has therefore confirmed that FFPP sections have diagnostic potential provided that researchers are aware of the biochemical changes due to tissue processing highlighted by this study.
\end{abstract}

Keywords: Raman; Fourier transform infrared spectroscopy; Fresh tissue; Frozen sections; Formalin-fixed paraffin processed sections; Tissue processing; Fixation; Wax; Cancer 


\section{Introduction}

In the past number of years many studies have been carried out using vibrational spectroscopy to classify tissue with a view to cancer diagnosis [1]. Both IR and Raman spectroscopy have been employed. Raman spectroscopy is very similar to the more frequently used Fourier transform infrared (FT-IR) spectroscopic technique and both spectroscopic techniques are very complementary. Vibrations that are strong in an infrared spectrum, those involving strong dipole moments, are usually weak in a Raman spectrum. Likewise, those non-polar vibrations that give very strong Raman bands usually result in weak infrared signals. For example, hydroxyl or amine stretching vibrations and the vibrations of carbonyl groups, are usually very strong in an FT-IR spectrum and usually weak in a Raman spectrum. However, the stretching vibrations of carbon-carbon double or triple bonds and symmetric vibrations of aromatic groups are very strong in the Raman spectrum. Raman however, has the advantage of minimal interference from water so is a good choice for biological samples with a view to in vivo measurements. Some of the tissue types examined by various groups include; cervical [24], breast [5-7], skin [8-12], lung [13], brain [14,15] bladder [16], esophagus [17-19], colon [20], liver [21,22], thyroid [23] and prostate [24,25]. A variety of different methods of sample preparation have been employed in these studies, such as; fresh, frozen, airdried, formalin-fixed and de-waxed formalin fixed paraffin preserved (FFPP) tissue sections. The overwhelming majority of tissue studies have been carried out on either frozen tissue or de-waxed FFPP sections [1]. Although considered the gold-standard, frozen tissue is difficult to obtain, deteriorates rapidly and cannot provide retrospective analysis. However, there is a plentiful supply of archival tissue samples, preserved and mounted in wax blocks. Due to the prevalence of these FFPP tissue sections, a better understanding of the effects of processing could unlock the potential diagnostic capabilities of these sections. The sections go through many processing steps before spectroscopic evaluation. If these processing steps result in changes in bands identified as diagnostically significant, this would have implications for tissue classification and could compromise the potential of these FFPP sections in a diagnostic capacity. Previous studies using vibrational spectroscopy have been carried out into the effect of formalin fixation as well as ex vivo handling [26-28]. Huang et al. [26] investigated the effects of formalin fixation on human bronchial tissue using Raman spectroscopy. A decrease in overall Raman intensities was observed and notable formalin peaks were identified at 907, 1041 and $1492 \mathrm{~cm} \_1$. Shim and Wilson [27] examined the effect of tissue drying, formalin fixation and snap freezing on normal hamster tissues using Raman spectroscopy. The study found that formalin did not contaminate the Raman spectrum (with the exception of the $1041 \mathrm{~cm} \_1$ band), however drying/ dehydration was found to disrupt protein vibrational modes. FT-IR spectroscopy was used by Pleshko et al. [28] to study the effects of fixation in ethanol and formalin, as well as methacrylate embedding. The study found ethanol fixation of fetal rat bone tissue resulted in changes in the Amide I and II bands (1650 and $1550 \mathrm{~cm} \_1$, respectively), a result of alteration of the protein conformation of the tissue. This study seeks to re-examine the above and extend the study to investigate the effect of xylene, paraffin wax embedding and subsequent de-waxing on human tissue using both Raman and FT-IR spectroscopy. The use of both techniques on the same samples maximises the amount of structural information obtained from the 
tissue. The steps involved in processing these sections can be summarised as follows: tissue excising, fixation in formalin, tissue dehydration in alcohol, embedding in paraffin wax, microtomy and removal of wax. This study examined the biochemical structure of normal parenchymal tissue from the placenta at each of these processing steps using both Raman and IR spectroscopy. This tissue was chosen due to its homogenous nature. This minimises the likelihood of recording spectra from different cell types, which would be expected to produce different spectral features. A homogenous tissue ensures that it is only the effects of processing that are being detected, rather than point-to-point inhomogeneities. The effect of tissue freezing was also examined and compared to freshly excised tissue.

\section{Experimental}

\subsection{Raman spectroscopy}

An Instruments S.A. Labram Raman spectroscopic confocal microscope was used, with an Argon ion laser operating at a wavelength of $514.5 \mathrm{~nm}$. The Labram imaging system is a fully confocal Raman microscope system, with a motorised XY sample stage for automated Raman imaging. The system includes a stigmatic spectrometer with two motorised gratings, of which the 1800 grooves $/ \mathrm{mm}$ grating was used. The resolution of the system operating with the 1800 grooves/mm was $1.65 \mathrm{~cm} \_1 /$ pixel. Detection of the scattered light was performed using a Peltier cooled, 16 bit dynamic range CCD detector with 1024 _ 256 pixels. A 50_ objective lens was used. The laser power at the sample was measured and found to be about $6.5 \_0.05 \mathrm{~mW}$, focused to a spot size of $2 \mathrm{~mm}$ at the tissue surface. The focal depth and thus the maximum sampling thickness is about $2 \mathrm{~mm}$. The scattered Raman signal was integrated for 60-150 s and measured over a spectral range of 400-1900 cm_1 with respect to the excitation frequency. Once acquired each spectrum was baseline corrected, noise subtracted and lightly filtered using a third order linear model to improve clarity.

\subsection{FT-IR spectroscopy}

A Perkin-Elmer Spectrum GX single-beam, Michelson interferometer-based, Fourier transform infrared spectrometer was used. The spectrometer has a dual level optical module that is sealed and desiccated. The radiation source was provided by a built-in 35 $\mathrm{W}$ tungsten halogen illuminator. The medium beam MCT detector covers the range from 5500 to $550 \mathrm{~cm} \_1$. MIR and FIR beam splitters and DTGS detector kits allow the range $7000-50 \mathrm{~cm} \_1$ to be covered. The range was set to $4000-400 \mathrm{~cm} \_1$ with a resolution of 8 cm_1. The spectrometer was configured with the Auto IMAGE microscope system, which was operated in reflectance mode. The aperture was set to $50 \mathrm{~mm} \_50 \mathrm{~mm}$, with a gain of 4. Each sample was scanned for 128 scans, baseline corrected and lightly smoothed.

\subsection{Sample preparation}


Normal parenchymal tissue from the placenta was obtained from the National Maternity Hospital, Holles Street, Dublin. Tissue was examined at each stage of the fixation, embedding and de-waxing processes. Tissue was divided into six different pieces and was examined as follows:

1. Fresh tissue: tissue was refrigerated and examined within $24 \mathrm{~h}$. The tissue was crosssectioned and a narrow section from the tissue centre was excised using a scalpel. The tissue was then rinsed in phosphate buffered saline (PBS), before being mounted onto silver oxide MirrIR slide (MirrIR, Kevley Technologies, Chesterland, OH, U.S.) for examination.

2. Frozen tissue: tissue was placed on a metal chuck with optimal cutting temperature (OCT) as support medium and then placed in a cryostat at _20 8C. Tissue was sectioned into $10 \mathrm{~mm}$ slices using the cryostat's internal microtome and mounted onto silver oxide MirrIR slides. Tissue spectra were recorded when the tissue thawed.

3. Formalin-fixed tissue: tissue was placed in formalin for $24 \mathrm{~h}$. The tissue was cross sectioned and a narrow section from the tissue centre was excised using a scalpel, rinsed in PBS, and mounted onto MirrIR slides before examination.

4. Formalin-fixed and dehydrated using xylene (Serosep, Limerick, Ireland): tissue was automatically processed through formalin and xylene. Again the tissue was crosssectioned and a piece of tissue was rinsed in PBS before being mounted on the silver oxide slides.

5. FFPP tissue mounted in wax: tissue was automatically processed to wax as follows:

i. vacuum fixed in $10 \%$ buffered formal saline histograde $\mathrm{pH}$ 6.8-7.2 (J.T. Baker, Deventer, The Netherlands) and heated to $358 \mathrm{C}$;

ii. vacuum dehydration in industrial methylated spirit IMS T100 (Lennox, Dublin, Ireland) heated to $358 \mathrm{C}$;

iii. vacuum clearing in xylene (Serosep, Limerick, Ireland) and heated to $358 \mathrm{C}$;

iv. vacuum impregnation with Tissue Tek III Embedding Wax with polymer added (Sakura, Zoeterwoude, The Netherlands) and heated to 59 8C. $10 \mathrm{~mm}$ sections were sliced using a microtome and mounted on MirrIR slides.

6. De-waxed FFPP sections: $10 \mathrm{~mm}$ waxed sections were immersed in a series of baths consisting of two baths of xylene (BDH, Dorset, UK) for 5 min and 4 min, respectively, two baths of ethanol absolute (Merck, Dorset, UK) for 3 min and $2 \mathrm{~min}$, and a final bath of Industrial Methylated Spirits 95\% (Lennox, Dublin, Ireland) for $1 \mathrm{~min}$.

Each category investigation was carried out on 3 parallel tissue pieces or sections within the category and a minimum of 10 spectra were recorded from each using both IR and Raman spectrometers. A representative selection is shown.

\section{Results}

Fig. 1 compares the Raman spectra of fresh tissue and frozen tissue. Both tissues produce good spectra. There is an overall deterioration in the spectrum of the frozen tissue section compared to the fresh tissue. The most noticeable differences between the fresh and frozen tissue is the reduction in the intensity the $1002 \mathrm{~cm} \_1$ (C-C aromatic ring stretching), $1447 \mathrm{~cm} \_1$ ( $\mathrm{CH} 2$ bending mode of proteins and lipids) and $1637 \mathrm{~cm} \_1$ (Amide I band). The frozen tissue also displays an additional contribution at $1493 \mathrm{~cm} \_1$. Fig. 2 compares the Raman spectra of fresh tissue with formalin fixed tissue and tissue 
fixed and soaked in xylene. The most obvious change in the tissue with the fixation in formalin is the dramatic reduction in the intensity of the Amide I band (1637 cm_1) and the addition of the peak at $1490 \mathrm{~cm} \_1$. Soaking in xylene prior to wax embedding creates significant spectral differences in the tissue. Strong peaks appear at 620, 1002, 1032 and $1601 \mathrm{~cm} \_1(\mathrm{C}-\mathrm{C}$ twist aromatic ring, $\mathrm{C}-\mathrm{C}$ stretching aromatic ring, $\mathrm{C}-\mathrm{C}$ skeletal stretch and $\mathrm{C}-\mathrm{C}$ in plane bending, respectively). The addition of the small peak at $1203 \mathrm{~cm} \_1$ is attributed to the $\mathrm{C}-\mathrm{C} 6 \mathrm{H} 5$ stretch mode. There is also a reduction in the $1585 \mathrm{~cm} \_1$ band (C C olefinic stretch), and the Amide I band has also reappeared at $1637 \mathrm{~cm} \_1$. Fig. 3 compares the Raman spectra of fresh tissue with FFPP sections in wax and de-waxed FFPP sections using xylene as a de-waxing agent. In 3(B) the wax contributions are clearly seen at $1063,1130,1296$ and $1436 \mathrm{~cm} \_1$ (C-C skeletal stretch random conformation, $\mathrm{C}-\mathrm{C}$ skeletal stretch transconformation, $\mathrm{CH} 2$ deformation, and $\mathrm{CH} 2$ scissoring, respectively). It is clear from Fig. 3C that all the wax is not being removed and residualwax peaks are present in the tissue after de-waxing. Also as seen in the previous processing steps, there is an overall reduction in signal from the biological material after freezing and formalin fixation.

Fig. 4 examines the FT-IR spectra of fresh and frozen tissue. The frozen tissue displays an overall reduction in intensity. Also, the Amide I and II bands (1673 and $1554 \mathrm{~cm} \_1$, respectively) are shifted indicating changes in protein conformation in the frozen tissue section. Fig. 5 examines the effect of formalin fixation and formalin and xylene processing before wax embedding using FT-IR spectroscopy. There is an apparent 10 cm_1 shift in the Amide I and II bands after formalin fixation. The reduction in intensity of the Amide I band seen using Raman spectroscopy is mirrored in the FT-IR spectra. Xylene results in an increase in intensity of the $\mathrm{CH} 2$ scissoring (1454 cm_1) and amide III vibrations $\left(1239 \mathrm{~cm} \_1\right)$. A reduction in the intensity of the $1398 \mathrm{~cm} \_1$ band (C O symmetric stretch) is observed after fixation. Fig. 6 compares the FT-IR spectra of fresh tissue with wax embedded tissue and de-waxed FFPP sections. The wax peak is clearly visible in Fig. 6B at 1465 cm_1 (CH2 scissoring). The Amide I band has shifted back to its original position (1673 cm_1) after de-waxing Fig. 6C. Again there is a loss in signal intensity after de-waxing.

\section{Discussion}

The Raman spectrum of frozen tissue compared to fresh tissue displays a reduction in intensity of the $1002 \mathrm{~cm} \_1$ (C-C aromatic ring stretching), $1447 \mathrm{~cm} \_1$ ( $\mathrm{CH} 2$ bending mode of proteins and lipids) and $1637 \mathrm{~cm} \_1$ (Amide I) bands. This overall reduction in Raman intensities was also observed by Huang et al. [26]. A new peak was also observed at $1493 \mathrm{~cm} \_1$. In order to eliminate the mounting medium OCT as a possible contributing factor, Raman spectra were recorded from OCT, which had no Raman signal at 1493 $\mathrm{cm} \_1$ and therefore, was not the cause of the additional peak. The effects of freezing on cells has been well documented in the cryogenics field and it is well known that a reduction in temperature can result in depolymerisation of the cellular cytoskeleton [29]. The cellular cytoskeleton is composed of different types of protein fibres. Any depolymerisation of these proteins would result in unraveling of the secondary structure and hence an increase in the $\mathrm{NH} 3+$ and $\mathrm{COO}_{-}$vibrations. The Raman frequencies for 
vibrations involving these groups occur between $1485-1550 \mathrm{~cm} \_1$ and $1560-1600 \mathrm{~cm} \_1$ respectively, and both increase after the tissue had been frozen. Thus the appearance of the band at $1493 \mathrm{~cm}_{-} 1$ can be attributed to an increased contribution from NH3+ deformation, as a result of protein structural changes on freezing tissues. Fixation in formalin also produced a reduction in intensity of the Amide I band at $1637 \mathrm{~cm} \_1$. Although, all the effects of formalin fixation are not completely understood, the general principles are known. Aldehydes in formalin form cross-links between proteins creating a gel, thus retaining cellular constituents in their in vivo relationship to each other. Soluble proteins become fixed to structural proteins. The majority of cross-links are formed between the nitrogen atom of lysine and the nitrogen atom of a peptide linkage (Fig. 7). This cross-link (methylene bridge) alters the amide from a secondary amide to a tertiary amide, which in turn alters the frequency of the $\mathrm{C} \mathrm{O}$ vibration, which could explain the loss of the band $1637 \mathrm{~cm} \_1$. The new peak at $1490 \mathrm{~cm} \_1$ may be due to protein unravelling, resulting in the increased activity of the $\mathrm{NH} 3+$ deformation similar to that seen after tissue freezing. Alternatively, a study by Wojciechowski et al. [30] found a distinct Raman band at $1491 \mathrm{~cm} \_1$ which was assigned to the $\mathrm{C}-\mathrm{N}$ stretching vibration coupled with the in-plane $\mathrm{C}-\mathrm{H}$ bending in amine radical cations. Such coupled vibrations may be present in the methylene bridging system proposed to form after formalin fixation (Fig. 7). It can be assumed that there is no residual formalin due to the fact that other characteristic formalin peaks at 907 and $1041 \mathrm{~cm}_{-} 1$ (Huang et al. [26]) are not present, and any excess formalin has been removed by rinsing in PBS. In Fig. 2C, xylene contributions are clear at $620,1002,1032,1203$ and $1601 \mathrm{~cm} \_1$. These $\mathrm{C}-\mathrm{C}$ ring vibrations are to be expected given the aromatic structure of xylene. The absence of the $1490 \mathrm{~cm} \_1$ band associated with the presence methylene bridges suggested above, and the re-appearance of the Amide I band suggests that xylene has reversed some of the cross-links created with formalin fixation, and the amide I band has reverted to a secondary amide as seen in fresh tissue $\left(1637 \mathrm{~cm} \_1\right)$. The xylene signature peaks are not present in the de-waxed tissue (Fig. 3) so it may be assumed that all xylene is being removed after de-waxing. However, it is clear from the Raman spectrum of de-waxed FFPP sections that all the wax isnot being removed. Strong wax contributions were seen at $1063,1130,1296$ and $1436 \mathrm{~cm} \_1$, so effective wax clearing is an issue when dealing with FFPP sections.

Using FT-IR spectroscopy, fresh tissue provided the most detailed spectra, whereas freezing resulted in loss of intensity and a shift in the Amide I and II bands. This shifting is assumed to result from the de-polymerisation outlined previously. Formalin fixation produced a shift of $10 \mathrm{~cm} \_1$ in the amide I and II bands, which corroborates the findings of Pleshko et al. [28] who found the amide bands shifting when fixed in ethanol. Freezing and formalin fixation lead to a large reduction in the intensity of the $1398 \mathrm{~cm} \_1$ band (C $\mathrm{O}$ symmetric stretch), which corroborates the Raman findings. Xylene resulted in an increase in the intensity of the 1239 and $1454 \mathrm{~cm} \_1$ bands, but did not display the dramatic spectral differences seen using Raman spectroscopy. The FT-IR signature contribution of wax at $1465 \mathrm{~cm} \_1$ was also visible. After de-waxing there is yet another reduction in intensity. The Amide I band shifted back to its original position of 1673 $\mathrm{cm}_{-} 1$ as seen in fresh tissue and the wax signature at $1465 \mathrm{~cm} \_1$ has been reduced. However it is very difficult to say whether or not the wax has been removed using FT-IR spectroscopy. Again, in this instance Raman spectroscopy provides more detailed 
biochemical information. Therefore the $1454 \mathrm{~cm} \_1$ band ( $\mathrm{CH} 3$ asymmetric deformation) seen in fresh tissue and dewaxed FFPP sections, could easily be contaminated with contributions from wax occurring at $1465 \mathrm{~cm} \_1$. For this reason it is not advisable to use this peak in a diagnostic capacity. The reduction in overall signal intensity after processing seen in both Raman and FT-IR remains unexplained. The spectra of fresh, formalin fixed tissue were recorded from tissue pieces mounted on MirrIR slide, and therefore would inherently have variations in sample thickness. However, any variation in sample thickness would not have an effect on the intensity of the Raman spectra. This is due to the fact that Raman spectroscopy is a scattering effect, with a penetration depth of about $2 \mathrm{~mm}$, far below the minimum sample thickness of about $10 \mathrm{~mm}$. Sample thickness would affect the overall FT-IR signal intensity, due to the double transmission nature of the measurement. But the fact that this signal reduction was also seen in Raman confirms that the effect results from more than sample thickness variation. It is possible however, that differences in sample density between tissue sections and excised tissue pieces may account for the differences in overall signal intensity observed between samples. Although, de-waxed FFPP sections display a reduction in intensity, all of the bands in fresh tissue are present and many are diagnostically useful.

Generally speaking it was found that Raman spectroscopy produced more detailed spectra, and hence appears more sensitive to changes in tissue composition than FT-IR spectroscopy. Naturally, Raman spectroscopy is intrinsically more sensitive to some bands than FT-IR and vice versa. However, it is the higher spectral and spatial resolution that results in the more detailed spectra obtained using Raman spectroscopy. The spectral resolution of the Raman spectrometer $\left(2 \mathrm{~cm} \_1\right)$ is superior to the $8 \mathrm{~cm} \_1$ resolution used with the FT-IR spectrometer. Also, the much larger sampling area of the FT-IR spectrometer (although beneficial for sampling large areas) will also have the effect of averaging over many cells, broadening bands with the loss of spectral information. Studies carried out by our group [31], have shown significant differences between normal and malignant tissue using de-waxed FFPP sections. Many biochemical changes occur in regions outside those highlighted by this paper as affected by processing. A more complete study has already been performed into improvements in the de-waxing protocol [32]. The study examined many different methods of wax removal and found that the use of hexane instead of xylene resulted in a far superior level of wax removal.

In summary, although bearing a close resemblance to fresh tissue, processed tissue has undergone many biochemical changes as shown. This systematic study has provided spectra at each of the processing stages involved in de-waxed FFPP sections. This gives a real insight into the biochemical changes resulting from each process. It has highlighted changes that would otherwise be unseen when comparing fresh with de-waxed FFPP sections. This study has confirmed that de-waxed FFPP sections can be diagnostically useful as they retain sufficient biochemical similarity to fresh tissue. However, researchers in this field should be aware of the spectral contributions affected by processing, particularly when utilising automated analysis techniques.

Acknowledgements 
Authors would like to express sincere thanks to all the staff in the Histology Department in the NMH Holles Street for all their generous co-operation. Thanks also to Paddy Lynch for his assistance with ChemSketch. The Focas Institute is funded under the National Development Plan 2000 to 2006 with assistance from the European Regional Development Fund.

\section{References}

[1] R. Dukor, Handbook of Vibrational Spectroscopy, vol. 5, 2002.

[2] U. Utzinger, A. Mahadevan-Jansen, D. Hinzelman, M. Follen, R Richards-Kortum, Appl. Spectrosc. 55 (8) (2001) 955.

[3] A.M.F.M. Mahadevan-Jansen, N. Ramanujam, A. Malpica, S. Thomsen, U. Utzinger, R. Richards-Kortum, Photochem. Photobiol. 68 (1) (1998) 123-132.

[4] Y.R.N. Yazdi, R. Lotan, K. Kline, M.F. Mitchell, W. Hittelman, R.R. RichardsKortum, Appl. Spectrosc. 53 (1) (1998) 82-85.

[5] N. Shah, A. Cerussi, C. Eker, J. Espinoza, J. Butler, J. Fishkin, R. Hornung, B. Tromberg, Proc. Natl. Acad. Sci. 98 (8) (2001) 4420-4425.

[6] J. Kneipp, T.B. Schut, M. Kliffen, M. Menke-Pluijmers, Puppels G, Vib. Spectrosc. 32 (1) (2003) 67-74.

[7] J. Smith, C. Kendall, A. Sammon, J. Christie-Brown, N. Stone, Technol. Cancer Res. Treat. 2 (4) (2003) 327-331.

[8] L.M. McIntosh, M. Jackson, H.H. Mantsch, J.R. Mansfield, A.N. Crowson, J.W.P. Toole, Vib. Spectrosc. 28 (1) (2002) 53-58.

[9] L.D. Nunes, A.A. Martin, L. Silveira, M Zampieri, Spectrosc.-An Int. J. 17 (2/3) (2003) 597-602.

[10] A. Nijssen, T.C. Bakker Schut, F. Heule, P.J. Caspers, D.P. Hayes, M.H.A. Neumann, G.J. Puppels, J. Invest. Dermatol. 119 (1) (2002) 64-69.

[11] P.J. Caspers, G.W. Lucassen, E.A. Carter, H.A. Bruining, G.J. Puppels, J. Invest. Dermatol. 116 (3) (2001) 434-442.

[12] M. Gniadecka, H.C. Wulf, O.F. Nielsen, D.H. Christensen, J Hercogova, Photochem. Photobiol. 66 (4) (1997) 418-423.

[13] S. Kaminaka, H.I.T. Yamazaki, E. Kohda, H. Hamaguchi, J. Raman Spectrosc. 32 (2001) 139-141.

[14] A. Mizuno, K. Hitajima, K. Kawauchi, S. Muraishi, Y. Ozaki, J. Raman Spectrosc. 25 (1) (1994) 25-29.

[15] S. Keller, B. Schrader, A. Hoffmann, W. Schrader, K. Metz, A. Rehlaender, J. Pahnke, M. Ruwe, W Budach, J. Raman Spectrosc. 25 (7-8) (1994) 663-671.

[16] P. Crow, N. Stone, C. Kendall, J. Uff, A. Ritchie, M. Wright, J. Urol. 169 (4) (2003) 871, Suppl. S.

[17] I.A. Boere, T.C.B. Schut, J. van den Boogert, R.W.F. de Bruin, G.J. Puppels, Vib. Spectrosc. 32 (1) (2003) 47-55.

[18] C. Kendall, N. Stone, N. Shepherd, K. Geboes, B.Warren, R. Bennett, H Barr, J. Pathol. 200 (5) (2003) 602-609.

[19] L.M.W.K. Song, T.Y. Gao, A. Molckovsky, L. Burgart, T. Smyrk, N. Buttar, L. Lutzke, B. Wilson, K. Wang, Gastrointest. Endosc. 57 (5) (2003) AB91.

[20] A. Molckovsky, L.M.W.K. Song, M.G. Shim, N.E. Marcon, B.C. Wilson, Gastrointest. Endosc. 57 (3) (2003) 396-402. 
[21] L. Chiriboga, M. Yee, M. Diem, Appl. Spectrosc. 54 (1) (2000) 1-8.

[22] S.R. Hawi, W.B. Cambell, A. Kajdacsy-Balla, R. Murphy, F. Adar, K. Nithipatikom, Cancer Lett. 110 (1996) 35-40.

[23] K.Z. Liu, C.P. Schultz, E.A. Salamon, A. Man, H.H. Mantsch, J. Mol. Struct. 661 (2003) 397-404.

[24] P. Crow, C. Kendall, J. Uff, H. Gilbert, R. Persad, M. Wright, J. Urol. 169 (4) (2003) 1618, Suppl. S.

[25] C. Paluszkiewicz, W.M. Kwiatek, J. Mol. Struct. 565 (2001) 329-334.

[26] Z.W. Huang, A. McWilliams, S. Lam, J. English, D.I. McLean, H. Lui, H. Zeng, Int. J. Oncol. 23 (3) (2003) 649-655.

[27] M.G. Shim, B.C. Wilson, Photochem. Photobiol. 63 (5) (1996) 662-671.

[28] N.L. Pleshko, A.L. Boskey, R. Mendelsohn, Calcified Tissue Int. 51 (1) (1992) 72 77.

[29] P.F. Watson, G.J. Morris, Symposia of the Society for Experimental Biology, vol. 41, Company of Biologists, Cambridge, UK, 1987, pp.311-340.

[30] P.M. Wojciechowski, W. Zierkiewicz, D. Michalska, P. Hobza, J. Chem. Phys. 118 (24) (2003) 10900-10911.

[31] E. O ' Faola'in, J. Conroy, M. Hunter, P. Kelehan, H. Lambkin, C. Mothersill, H.J. Byrne, F.M. Lyng, Proc. SPIE 4876-18 (2003) 84-91.

[32] E.O’ . Faola'in, M.B. Hunter, J.M. Byrne, P. Kelehan, H.A. Lambkin, H.J. Byrne, F.M. Lyng, J. Histochem. Cytochem. 53 (2005) 121-131 